\section{$5 / 12.9798(2)$}

PPPL-2846

UC-420,427
PREPARED FOR THE U.S. DEPARTMENT OF ENERGY, UNDER CONTRACT DE-AC02-76-CHO-3073

THE INTERACTION OF ENERGETIC ALPHA-PARTICLES WITH INTENSE LOWER HYBRID WAVES

BY

N.J. FISCH AND J.M. RAX

June, 1992

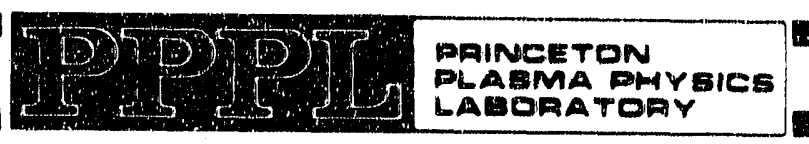
plasma phy bies
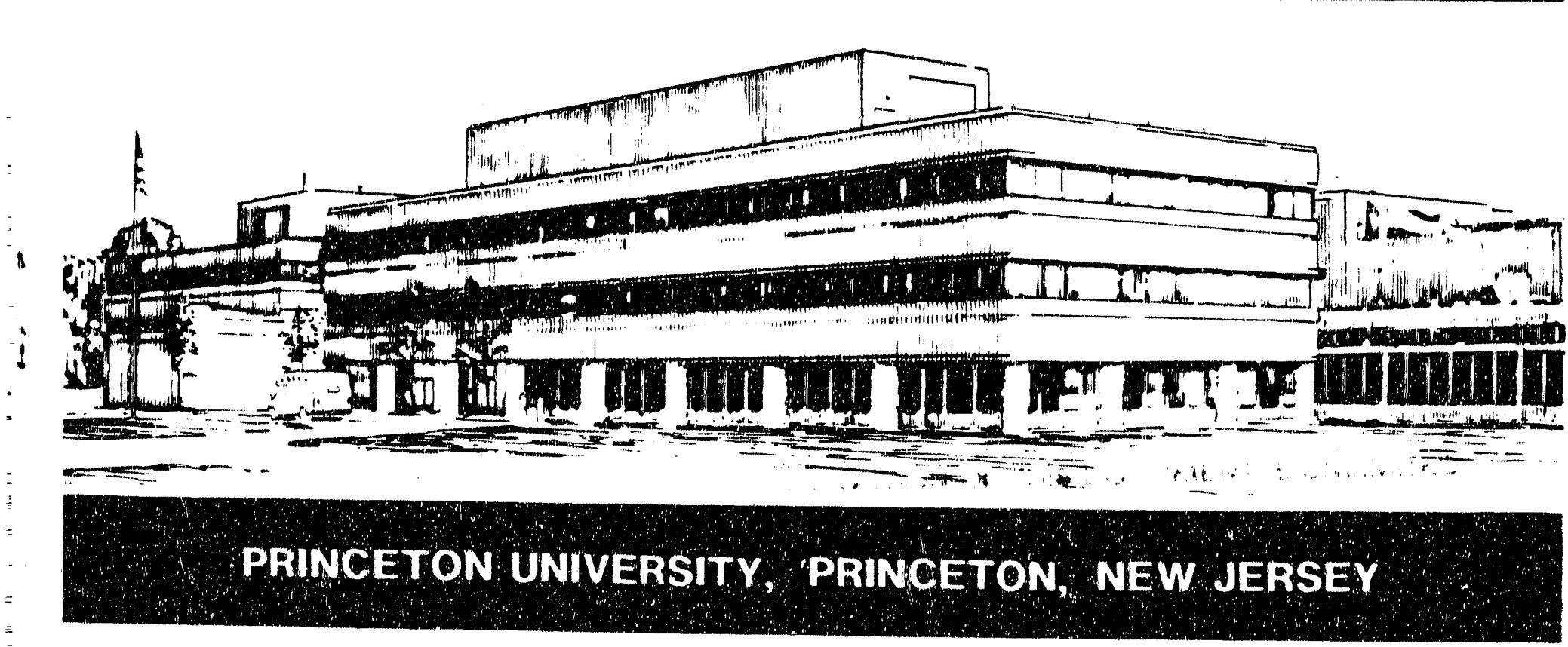


\section{NOTICE}

This report was prepared as an account of work sponsored by an agency of the United States Government. Neither the United States Government nor any agency thereof, nor any of their employees, makes any warranty, express or implied, or assumes any legal liability or responsibility for the accuracy, completeness, or usefulness of any information, apparatus, product, or process disclosed, or represents that its use would not infringe privately owned rights. Reference herein to any specific commercial produce, process, or service by trade name, trademark, manufacturer, or otherwise, does not necessarily constitute or imply its endorsement, recommendation, or favoring by the United States Government or any agency thereof. The views and opinions of authors expressed herein do not necessarily state or reflect those of the United States Government or any agency thereof.

\section{NOTICE}

This report has been reproduced directly from the best available copy.

Available to DOE and DOE contractors from the:

Office of Scientific and Technical Information

P.O. Box 62

Oak Ridge, TN 37831 ;

Prices available from (615) 576-8401.

Available to the public from the:

National Technical Information Service

U.S. Department of Commerce 5285 Port Royal Road

Springfield, Virginia 22161

703.487 .4650 


\title{
The Interaction of Energetic Alpha-Particles WITH INTENSE LOWER HYBRID WAVES
}

\author{
Nathaniel J. Fisch and Jean-Marcel Rax ${ }^{\dagger}$ \\ Princeton Plasma Physics Laboratory \\ PPPL--2846 \\ Princeton University, Princeton, NJ 08543 \\ DE92 016805
}

\begin{abstract}
Lower hybrid waves are a demonstrated, continuous means of driving toroidal current in a tokamak. When these waves propagate in a tokamak fusion reactor, in which there are energetic $\alpha$-particles, there are conditions under which the $\alpha$-particles do not appreciably damp, and may even amplify, the wave, thereby enhancing the current-drive effect. Waves traveling in one poloidal direction, in addition to being directed in one toroidal direction, are shown to be the most efficient drivers of current in the presence of the energetic $\alpha$-particles.
\end{abstract}

† permanent address: Centre d'Études Nucléaires de Cadarache, Association ECRATOM-CEA Sur la Fusion Contrôlée, Saint-Paul-lez-Durance, France. 
Either for heating the fuel ions or for driving current, delivering power to the tokamak is a central problem in tokamak plasma research, and an effective means of delivering this power is through the lower hybrid wave. These waves, directed into the plasma by means of an array of waveguides, may be absorbed either by electrons or ions. These waves are also an efficient ineans for steady-state current drive when they are absorbed largely by electrons traveling in one toroidal direction. ${ }^{1}$ Unfortunately, in the most favorable wave regimes in a tokamak reactor, the current-drive efficiency may be reduced greatly because these waves tend also to be absorbed by energetic $\alpha$-particles. ${ }^{2}$

For heating by intense waves, the $\alpha$-particles tend to absorb more wave power than $\mathrm{dc}$ the electrons, because the electron interaction exhibits quasilinear saturation, i.e., the electron velocity distribution flattens in the region where the wave phase velocities are finite. The $\alpha$-particle interaction exhibits no saturation, because once in resonance, the $\alpha$-particle remains in resonance even as it gains energy. Recent calculations, ${ }^{3-5}$ which explore this severe damping by $\alpha$-particles, are made under the assumption that the plasma is infinite and homogeneous.

We show, however, that in an inhomogeneous plasma subjected to localized wave power, the interaction of $\alpha$-particles with lower hybrid waves can exhibit very different and useful effects. Of particular interest is the case of electrostatic waves with wavenumbers predominantly in the direction perpendicular to the magnetic field, but in the magnetic surface - for tokamaks with circular cross section, this would mean a large $k_{\theta}$, where $\hat{\theta}$ is the azimuthal direction, but a concentration of the wave amplitude in the radial direction. Such regimes are found in wave-tracing solutions that circle the tokamak center. ${ }^{6-8}$ The nature of the wave-particle interaction can then be qualitatively different: First, the $\alpha$-particle diffusion in velocity is now tied to diffusion in space. Thus, upon absorbing energy the $\alpha$-particle may be forced out of the wave region, saturating the absorption. Second, if there is a gradient in the $\alpha$-particle distribution, then there is even the possibility of wave amplification, rather than wave damping. Wave amplification by $\alpha$-particles could accompany wave damping by electrons, leading to very efficient current drive.

The energy tapped in wave amplification is the free-expansion energy in radially concentrated $\alpha$-particles. A similar tapping of free energy, involving lower hybrid waves, occurs in the so-called lower-hybrid drift instability, ${ }^{9-10}$ 
where the free energy arises from the drift of the ion species.

The consequences of tapping $\alpha$-particle energy, to the extent that it occurs, are very favorable for tokamak operation: the wave amplification and diffusive effect removes from the center unwanted helium ash, diminishes the $\alpha$-particle energy which makes for less energy available to fuel unwanted instabilities, and channels this energy to lower hybrid waves traveling in one toroidal direction to make more efficient the current drive. It is the purpose of this paper to identify and to quantify this effect.

To analyze these possibilities, we construct a Green's function for the energy exchange. We consider an electrostatic wave, with wavenumber in the $y$-direction, interacting with $\alpha$-particles magnetized in a strong $z$ directed magnetic field, $B$. The $\alpha$-particles are assumed to be produced with a gradient in the $x$-direction. These $\alpha$-particles are assumed to be magnetically confined in the plasma, but not in the wave, i.e., $k_{y} \rho_{\alpha} \gg 1$, where $\rho_{\alpha}$ is the $\alpha$-particle gyroradius. Thus, if, due to the wave, the $\alpha$ particle momentum changes by $m_{\alpha} \Delta v_{y}$, then the change in the $\alpha$-particle energy is $\Delta E=m_{\alpha} v_{y} \Delta v_{y}$, and the change in the gyrocenter is $\Delta x_{\mathrm{gc}}=$ $\Delta v_{y} / \Omega_{\alpha}$. where $\Omega_{\alpha}=2 \mathrm{eB} / \mathrm{m}_{\alpha}$ is the gyrofrequency. The wave-particle resonance is $u=k_{y} v_{y}$, so upon exchanging energy $\Delta E$ with the wave, the particle moves $\Delta x_{\mathrm{gc}}=\Delta E k_{y} / m_{\alpha} \Omega_{\alpha} \omega$. The excursions in gyroradius are proportional to the excursions in energy, but inversely proportional to the wave phase velocity: assuming wave phase velocities about 6 ion thermal relocities $v_{\mathrm{Ti}}$ (so as to avoid ion Landau damping), then, for a change in energy of $m_{\alpha} v_{\alpha}^{2} / 2=3.5 \mathrm{MeV}$ (where $v_{\alpha}$ is the birth speed), there would be a change in the guiding-center position of $L=v_{\alpha}^{2} k_{y} / 2 \omega \Omega_{\alpha}$, which might be about $20 \mathrm{~cm}$ in a $5 \mathrm{~T}$ magnetic field.

A diffusion equation in one dimension can be obtained by integrating the quasilinear diffusion equation for the $\alpha$-particles over parallel velocity space $^{11}$ to obtain

$$
\frac{\partial}{\partial \tau} F(\epsilon, X, t)=\frac{\partial}{\partial \epsilon} \epsilon F+\left(\frac{\partial}{\partial \epsilon}+\frac{\partial}{\partial X}\right) \frac{D(\epsilon, X)}{\sqrt{\epsilon-\epsilon_{w}}}\left(\frac{\partial}{\partial \epsilon}-\frac{\partial}{\partial X}\right) F+\frac{S}{\sqrt{1-\epsilon}},
$$

where $F$ is the $\alpha$-particle distribution integrated over parallel velocity, and where we have normalized $\epsilon=v_{\perp}^{2} / v_{\alpha}^{2}, X=x_{g c} / L, \tau=2 v t$, and $\epsilon_{w}=\left(\omega / k_{y} v_{n}\right)^{2}$. The first term on the right represents slowing down on electrons, the dominant collisional relaxation for energetic a-particles, 
where $\nu=16 \sqrt{2 \pi m_{e}} e^{4} n_{e} \ln \Lambda / 3 T_{e}^{3 / 2} m_{\alpha}$ is the slowing down rate of $\alpha$ particles on electrons. The third term is related to the $\alpha$-particle source, where $S(x)=\dot{N}(x) / 4 \pi v_{\alpha}^{2} \nu$, and where $\dot{N}$ is the $\alpha$-particle production rate per unit length. The second term gives the quasilinear diffusion of the $\alpha$ particles, where the diffusion coefficient may be written, under very general conditions, ${ }^{12}$ as

$$
D(\epsilon, X)= \begin{cases}\left(\omega / \nu \epsilon_{w}^{3}\right)\left(v_{o s c} / v_{\alpha}\right)^{2} & \text { if } \epsilon>\epsilon_{w} \text { and } 0 \leq X \leq A \\ 0 & \text { otherwise }\end{cases}
$$

where $v_{\text {osc }}=2 e E / m \omega$ would be the oscillating velocity of an $\alpha$-particle in the electrostatic field of strength $E$, and where $A$ is the normalized radial extent of the lower hybrid power.

To calculate the energy exchange, define the quantity $W\left(X_{0}, \epsilon_{0}, \tau\right)$, the (normalized) amount of energy absorbed from the waves, within time $\tau$, by an $\alpha$-particle with coordinates $\left(X_{0}, \epsilon_{0}\right)$ at $\tau=0$. Thus, for example, for quasilinear diffusion constant in time, the total (unnormalized) energy delivered to the waves, $\mathcal{E}_{u}$, within time $\tau$, can be written as

$$
\mathcal{E}_{w}(\tau)=\frac{\epsilon_{\alpha} L}{4 \nu} \int_{0}^{A} d X \int_{\epsilon_{w}}^{\infty} d \epsilon \int_{0}^{\tau} d \tau^{\prime} W\left(X, \epsilon, \tau-\tau^{\prime}\right) \frac{\dot{N}\left(X, \tau^{\prime}\right)}{\sqrt{1-\epsilon}}
$$

For comparison, the total $\alpha$-particle energy delivered to the plasma within time $\tau$ is simply

$$
\mathcal{E}_{\alpha}(\tau)=\frac{\epsilon_{\alpha} L}{2 \nu} \int_{0}^{A} d X \int_{0}^{\tau} d \tau^{\prime} \dot{N}\left(X, \tau^{\prime}\right)
$$

and the fraction of $\alpha$-particle energy delivered to the waves is $\mathcal{E}_{w}(\tau) / \mathcal{E}_{\alpha}$. This quantity, of course, could be negative in the case of absorption of wave power by the $\alpha$-particles.

Because we anticipate the use of high power density rf current-drive, it will be of particular interest to calculate $W$ in the limit $D \rightarrow \infty$. The high power density might arise naturally through focussing, or it might be incurred deliberately through brief, intense pulsing of the rf, which might improve the penetration ${ }^{13}$ and, under certain circumstances, even enhance the efficiency. ${ }^{14}$ In the limit $D \rightarrow \infty$, very quickly an $\alpha$-particle at $\left(X_{0}, \epsilon_{0}\right)$ 
will be diffused by the waves over the line $X+\epsilon=X_{0}+\epsilon_{0}$ (see Fig. 1). There will be an instantaneous energy exchange

$$
W_{0}\left(X_{0}, \epsilon_{0}\right)=\frac{\epsilon_{\max }+\epsilon_{\min }}{2}-\epsilon_{0}
$$

where the first quantity gives the final average kinetic energy over the diffusion region, and where the maximum energy is simply $\epsilon_{\max }=X_{0}+\epsilon_{0} . \Lambda$ diffusion path can be defined by $P(X, \epsilon) \equiv X+\epsilon$. For the minimum energy in the diffusion region, we have two separate cases

$$
\epsilon_{\min }=\left\{\begin{array}{lll}
P-A & \text { for } P>A+\epsilon_{w}, & \text { Region (b) } \\
\epsilon_{w} & \text { for } P<A+\epsilon_{w}, & \text { Region (a) }
\end{array}\right.
$$

which gives

$$
W_{0}\left(X_{0}, \epsilon_{0}\right)= \begin{cases}X_{0}-A / 2 & \text { for } P>A+\epsilon_{w} \\ \left(X_{0}-\epsilon_{0}\right) / 2+\epsilon_{w} / 2 & \text { for } P<A+\epsilon_{w}\end{cases}
$$

Note that the instantaneous energy exchange can be positive or negative; for $X_{0}$ small, it is always negative, which means that energy flows from the $\alpha$-particle to the wave, since, on balance, it is diffused by the wave to lower energy. This phenomenon, possible only when spatial diffusion is exploited, exhibits wave amplification! In contrast, the infinite homogeneous models of the wave plasma interaction (recovered here in the limit $L \rightarrow 0$ and, $\left.x_{0}, A \rightarrow \infty\right)$ allow diffusion in energy only. In such models, $W_{0}\left(X_{0}, \epsilon_{0}\right) \rightarrow$ $\infty$, since, in the limit $D \rightarrow \infty$, no normalizable time-asymptotic state exists.

After the instantaneous energy exchange, the particle density is uniform over the diffusion path, $X+\epsilon=P$, and no further energy exchange occurs until collisional slowing down renders the density no longer uniform over diffusion paths. The entire energy exchange is the sum of the instantaneous energy exchange and the subsequent energy exchange of the line, i.e.

$$
W\left(X_{0}, \epsilon_{0}, \tau\right)=W_{0}\left(X_{0}, \epsilon_{0}\right)+W_{\mathrm{L}}\left(P_{0}, \tau\right)
$$

where the line energy exchange depends through the initial coordinates on the initial line parameter $P_{0} \equiv P\left(X_{0}, \epsilon_{0}\right)$.

In calculating the energy exchange $W_{\mathrm{L}}\left(P_{0}, \tau\right)$ in the limit $D \rightarrow \infty$, first consider the case $P_{0}>A+\epsilon_{w}$, i.e., region (b). Imagine the slowing 
down and the diffusion occuring in separate, but nearly coincident steps: First, neglecting for an instant $\Delta \tau$ the wave diffusion, suppose all the $\alpha$ particles, initially distributed on the line $X+\epsilon=P_{0}$ slow down by means of collisions. Then, within the wave region $0 \leq X \leq A$, the $\alpha$-particles will become distributed uniformly over the collisionally decelerated line $X+$ $\psi \epsilon=P_{0}$, where $\psi=1+\Delta \tau$. Second, at the time $\Delta \tau$, suppose the waves instantaneously diffuse the $\alpha$-particles over the wave region, thus equalizing the density along the diffusion paths $X+\epsilon=$ constant. Here, zero-flux conditions are imposed at the boundaries at $X=0$ and at $X=A$, since the diffusion coefficient vanishes at the boundaries, and the slowing down is parallel to the boundaries. The energy exchange at time $\Delta \tau$ can then be found as the integral over the decelerated line density of the instantaneous energy exchange

$$
W_{\mathrm{L}}\left(P_{0}, \Delta \tau\right)=\int_{10}^{A} W_{0}\left(X,\left(P_{0}-X\right) / \psi\right) d X=0 .
$$

Remarkably, the energy exchange vanishes. This can be seen immediately from the fact that collisions, operating on a line of uniform density in region (b), leave unchanged the average $X$ as $A / 2$. Hence, by Eq.(6), there is no energy exchange.

The result of the slowing down and diffusion after the incremental time $\Delta \tau$ is to smear the $\alpha$-particles over the region $P_{\min } \leq P \leq P_{\max }$, where $P_{\min }=P_{0} / \psi$ and $P_{\max }=P_{0} / \psi+A(1-1 / \psi)$. If we approximate the smeared distribution as a line distribution at the average position $P=$ $P_{\mathrm{avr}}=\left(P_{\min }+P_{\max }\right) / 2$, then, setting $P_{1}=P_{\mathrm{avr}}$, the preceding analysis can be iterated to obtain the energy exchange in the next incremental instant of time. Clearly, repeated iteration results in no energy exchange until the line decelerates to region (a), which occurs, for some $P_{j}=A+\epsilon_{w}$, at time

$$
T_{a}=j \Delta \tau=\frac{P_{0}-A-\epsilon_{w}}{\Gamma_{u}}-A / 2 .
$$

The analysis here is equivalent to a multiple-time-scale approach in the limit $D \rightarrow \infty$, in which, on the shortest time scale, homogeneity along the diffusion paths is assured, and, on a longer time scale, the position of this path responds to the slowing down. 
The energy exchange in region (a) is more complicated; particles are no longer conserved in the wave region, since $\alpha$-particles leave the wave region through slowing down along the boundary $\epsilon=\epsilon_{w}$. Moreover, as we show, the energy exchange is nonvanishing. Suppose there is $\alpha$-particle density $N_{i}$, at time $i \Delta \tau$, along the line $X+\epsilon=P_{i}$ in region (a). The maximum value of $X$ along this line is $X_{i}=P_{i}-\epsilon_{w}$, occurring at the boundary $\epsilon=\epsilon_{w}$. If collisions operate now on this line for the incremental time $\Delta \tau$, then the result is a uniform line density over the line $X+\psi \epsilon=P_{i}$, with $X$, at the boundary $\epsilon=\epsilon_{w}$, now given by $P_{i}-\psi \epsilon_{w} \equiv X_{i}^{c}$, and the number of $\alpha$-particles lost from the region is evidently $N_{i}\left(X_{i}^{c}-X_{i}\right) / X_{i}$.

Upon diffusion: by waves, the average instantaneous energy exchange over the collisionally decelerated line at time $i \Delta \tau$ is

$$
\left\langle\Delta \mid V_{L}\right\rangle_{i}=\frac{1}{X_{i}^{c}} \int_{0}^{X_{i}^{c}} W_{0}\left(X,\left(P_{i}-X\right) / \psi\right) d X=\left(\frac{\psi-1}{4 \psi}\right)\left(P_{i}-\psi \epsilon_{w}\right) \text {, }
$$

where use was made of Eq.(6). The total energy exchange within time $\tau$, for a line originally at $P_{0}$ in region (a) can then be written as

$$
W_{L}\left(P_{0}, \tau\right)=\lim _{\Delta \tau \rightarrow 0} \sum_{i=0}^{\tau / \Delta \tau} N_{i}\left\langle\Delta W_{L}\right\rangle_{i}=\frac{1}{4} \int_{0}^{\tau} N\left(\tau^{\prime}\right)\left[P\left(\tau^{\prime}\right)-\epsilon_{w}\right] d \tau^{\prime} .
$$

For the diffusion of one particle, $N_{0}=N(0)=1$, and it remains now to find $N(\tau)$ and $P(\tau)$.

Let us again suppose that the main effect of slowing down and diffusion is to shift the line $X+\epsilon=P_{i}$ to $X+\epsilon=P_{i+1}$. Here, in region (a), we have $P_{\min }=P_{i} / \psi$ but $P_{\max }=P_{i}+(1-\psi) \epsilon_{w}$. Setting $P_{i+1}$ to the average position of the smeared distribution gives the difference equation

$$
P_{i+1}=P_{\mathrm{avr}}=P_{i}(1+\psi) / 2 \psi+(1-\psi) \epsilon_{w} / 2 .
$$

which, in the limit $\Delta \tau \rightarrow 0$, may be integrated to get

$$
P\left(\tau, P_{0}\right)=\left(P_{0}+\epsilon_{w}\right) \mathrm{e}^{-\tau / 2}-\epsilon_{w} .
$$

Using $N_{i+1}=X_{i}^{c} / X_{i}=\left(P_{i}-\psi \epsilon_{w}\right) /\left(P_{i}-\epsilon_{w}\right)$, and taking the limit $\Delta \tau \rightarrow 0$, we can write a differential equation for $N$ as

$$
\frac{d N(\tau)}{d \tau}=-\frac{\epsilon_{w}}{P(\tau)+\epsilon_{w}} N(\tau)
$$


and, using Eq.(13), we can solve

$$
N(\tau)=N_{0}\left(\frac{1-s \mathrm{e}^{\tau / 2}}{1-s}\right)
$$

where $s \equiv 2 \epsilon_{w} /\left(P_{0}+\epsilon_{w}\right)$. Note that both Eqs.(13) and (15) are valid until time $\tau=\tau_{\max }$, where $\tau_{\max }=2 \ln (1 / s)$, at which time $P \rightarrow \epsilon_{w}, N \rightarrow 0$, and the particles have all left the resonant region.

Plugging Eqs.(12) and (15), with $N_{0}=1$, into Eq.(11), we get,

$$
W_{L}\left(P_{0}, \tau\right)=\frac{1}{4} \frac{\epsilon_{w}}{1-s}\left\{-3 \tau-\frac{4}{s}\left(e^{-\tau / 2}-1\right)+2 s\left(e^{r / 2}-1\right)\right\} .
$$

Using Eqs.(6) and (16) in Eq.(7) gives the full Green's function for the energy exchange, through which the possibility of wave amplification can be examined.

First, note that $W_{L}$ is always positive, and, with increasing time, it becomes more positive. This means that, for no-flux boundary conditions, the amplification effect, if it occurs, is largest immediately. This suggests that brief pulses of $\mathrm{rf}$ may be more efficient than continuous ox excitation.

Second, consider the more likely case that the rf will be continuous, so that the asymptotic limit $\tau \rightarrow \tau_{\max }$ is appropriate in evaluating $W_{L}$. Note that all region (b) particles accumulate at time $T_{a}$ at region (a) at the point $P_{0}=\epsilon_{w}+A$. Thus, substituting for $\tau=\tau_{\max }$ and for $P_{0}$ in Eq.(16), and defining $3 \equiv 2 \epsilon_{w} / A$, we can write for all region (b) $\alpha$-particles

$$
W_{L}\left(P_{0}=\epsilon_{w}+A, \tau \rightarrow \infty\right)=\frac{A}{4}(3(\beta+1) \ln [\beta /(\beta+1)]+2 / \beta+3) .
$$

Consider two cases: For $\beta \rightarrow 0$, we have $W_{L} \rightarrow A / 2$. For $\beta \rightarrow \infty$, we have $W_{L} \rightarrow A / 83$. The first case is unfavorable for amplification, since $W_{L}$ is positive by at least the amount by which $W_{0}$ can be negative. The second case is less unfavorable, and, in fact, $W=W_{0}+W_{L}$ can be negative, implying amplification.

The physical explanation of the time-asymptotic energy exchange lies in exactly how the $\alpha$-particles leave the resonant region. Clearly, along any diffusion path, if a high energy particle leaves, it will be replaced, at expense to the wave, by low energy particles: on the other hand, if a low 
energy particle leaves, the reverse occurs, so that the wave is amplified. For the unfavorable case, $\beta \rightarrow 0, \alpha$-particles are never lost from the resonant region at the low energy boundary. This is because the low energy particles, with less perpendicular energy to lose, slow down less rapidly than do the high energy particles, and so tend to leave the diffusion path at $X=0$ rather than at $X=A$; for example, as $\epsilon_{w} \rightarrow 0$, the low energy particles are stuck in the resonant region and can only escape the wave diffusion through the diffusion path in which $P \rightarrow 0$. Thus, if particles are effectively lost in this limit only at $X=0$, the waves must, as found in Eq.(17), inject an average energy of $A / 2$. In the opposite limit, $\beta \rightarrow \infty$, high energy particles can, in fact, be lost from the resonant region at the boundary $X=0$, so the wave damping is less.

From this picture of the time-asymptotic energy exchange it becomes clear exactly how one must tamper with the no-flux boundary conditions in order to maximize the amplification effect. Suppose that there were an absorbing, rather than no-flux boundary; then, along any diffusion path that intersects the absorbing boundary, particles would be lost at that boundary. If the absorbing boundary can be placed either at $\epsilon=\epsilon_{w}$ or at $X=A$, then the $\alpha$-particles would be lost at the low energy end of the diffusion path, with net energy to the wave; e.g., in region (b), the energy recovery is simply $A / 2$.

How, precisely, to accomplish the desirable absorbing boundary conditions is beyond the scope of this letter. Note, however, that the physics of $\alpha$-particle transport is quite rich (see, e.g., Refs. 15-18), and one might consider a number of promising possibilities: for example, to accomplish the absorbing condition at $X=A$, a magnetic ripple might be applied to the outer flux surfaces of a tokamak, without producing significant interior ripple. Particles in the ripple, which might be maximized say at $X=A$, would be lost preferentially. In addition, there are interesting suggestions ${ }^{19}$ for introducing into a tokamak reactor certain kinds of magnetic turbulence to remove $\alpha$-particles preferentially at several hundred keV. Such an ash removal could produce an absorbing boundary near $\epsilon=\epsilon_{w}$.

The amount of energy extracted from the $\alpha$-particles can be significant. In principle, through the use of the energetically favorable boundary conditions, the available energy is the full perpendicular energy in a very strong $\alpha$-particle gradient, and about half this energy in a weak gradient. Suppose, for example, that about $5 \%$ of the fusion power output in a D-T 
reactor is to be recirculated for lower hybrid current drive, but that 10-20\% of the $\alpha$-particle power can be tapped. Since the $\alpha$-particle power is $20 \%$ of the fusion power, by utilizing the $\alpha$-particles, the circulating power can be held to only $1-3 \%$ of the power output, effectively cnhancing the efficiency of the current drive by a factor of 1.7-5! Since the cost of a reactor is very sensitive to the extent of recirculated power, the savings here could be important. Note also that in a $\mathrm{D}-\mathrm{He}^{3}$ reactor, in which the reaction products are protons, lower hybrid waves could similarly tap the energetic proton power, but with even greater effect, since the energetic protons dominate the total fusion power.

In summary, we have identified the importance of the poloidal momentum in lower hybrid waves when these waves interact with $\alpha$-particles. Choosing the right sense of the poloidal wavenumber, together with the right parallel wavenumber for current drive, can result in the channeling of free $\alpha$-particle energy to those lower hybrid waves capable of driving current. We identified the Green's function for the wave-particle energy exchange, and calculated, among other things, the important case of steady-state, intense excitation. We find that the effects described here, particularly when enhanced by certain means of ash removal, improve the prospects for economical, steady-state operation of a tokamak reactor.

\section{Acknowledgments}

This work was supported by the United States Department of Energy under contract number $\mathrm{DE}-\mathrm{ACO} 2-76-\mathrm{CHO} 3073$. 


\section{Figure Captions}

Fig. 1 The region of wave-particle interaction. The heavy solid line divides the interaction region into regions (a) and (b). The solid line terminating at $P_{0}$ is a typical diffusion path in region (a): after collisions lasting $\Delta \tau$, particles on the solid line $X+\epsilon=P_{0}$ slow down to the dotted line $X+(1+\Delta \tau) \epsilon=F_{0}$, which intersects the resonant region boundary at $X_{1}^{c}$.

\section{References}

1 N. J. Fisch, Phys. Rev. Lett. 59, 175 (1978).

2 K. M. Wong ard M. Ono, Nucl. Fusion 24, 615 (1984).

3 P. T. Bonoli and M. Porkolab, Nucl. Fusion 27, 1347 (1987).

4 E. Barbaro and F. Santini, Nucl. Fusion 31, 673 (1991).

5 M. Spada, M. Bornatici, and F. Engelmann, Nucl. Fusion 31, 447 (1991).

6 P. T. Bonoli, IEEE Trans. Plasma Sci. 12, 95.(1987).

7 E. Barbato, A. Cardinali and F. Santini, in Proc. Fourth Int. Symp.on Heating in Toroidal Plasmas (ed. H. Knoepfel and E. Sindoni. Rome, 1984), Vol. II, 1353.

8 E. Valeo and D. Eder. J. Comp. Phys. 27, 341 (1987).

9 R. C. Daridson, and ‥ T. Gladd, Phys. Fluids 18, 1327 (1975).

10 R. C. Daridson. Phys. Fluids 21. 1375 (1978).

$11 \mathrm{X} . J$. Fisch and J.-. . Rax, to appear in Nucl, Fusion (1992).

12 C.F. F. Karney Phys. Fluids 22, 2188 (1979).

13 R. H. Cohen. T. D. Roglien, P. T. Bonoli, and .I. Porkolab. Proc. 13th Int. Conf. on Plasma Physics and Controlled Nucl. Fusion Research (Washington, DC), (IAEA, Vienna, 1991), Vol. I p. 805.

14 N. J. Fisch, Rev. Mod. Phys. 59, 175 (1987).

$15 \mathrm{~V}$. Y. Goloborodko, Y. I. Kolesnischenko, and V A. Yavorskii. Physica Scriptia T16, 46 (1986).

16 G. Kamelander and D. J. Sigmar, Physica Scripta 45. 147 (1092).

17 R. B. White and H. E. Mynick. Phys. Fluids B 1980 (1989).

18 L. Chen. J. Vaclavik and G. W. Hammett, Vucl. Fusion 28389 (1988).

10 H. E. Mynick. in Proc. Int. Sherwood Fusion Theory Conf., abstract 1c18. Los Alamos Nat1. Lab. Report LA-LR-32-761 (1932). 


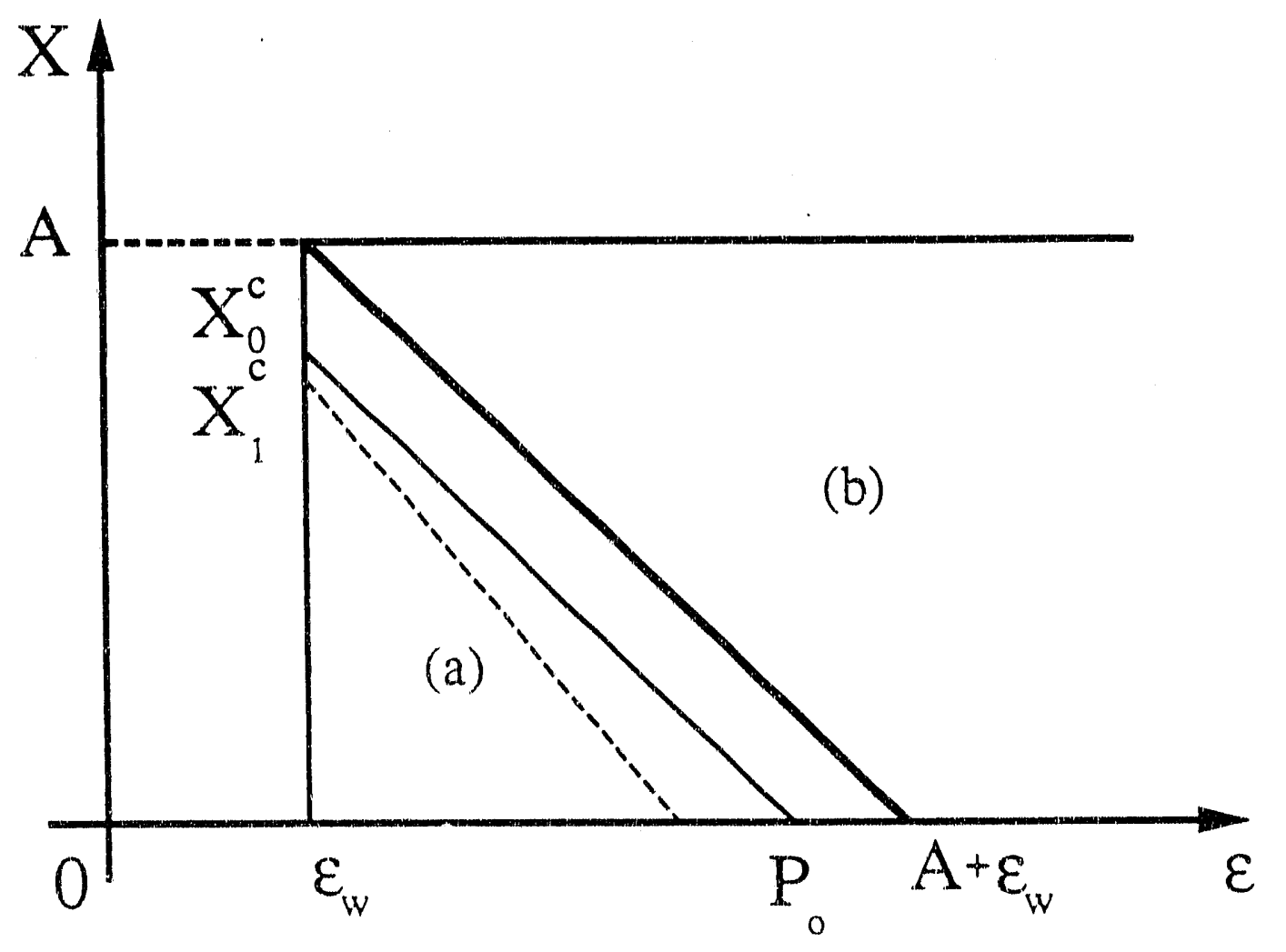

FIG. 1 
Dr. F. Peoloni, Univ of Wollongong, AUSTRALIA

Prot. M.H. Bremnan, Univ. of Sydnoy, AUSTRALIA

Plaema Rescearch Lob., Australion Nat. Univ., AUSTFALIA

Prot. I.A. Jonses, Flindors Univ. AUSTRMLLIA

Prot. F. Cep, Inst for Theoretical Physics, AUSTRIA

Prot. M. Hoindwer, Inativit for Theorswecto Phycik, AUSTRIA

Prot. M. Goosson, Astrowomican incituLt, BELGIUM

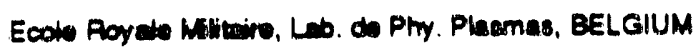

Commiceron-Europen, DG. XII.Fusion Prog., BELGIUM

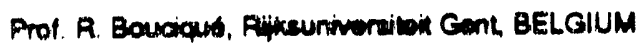

D. P.H. Sakenaka, Instuto Frica, BPUZIL.

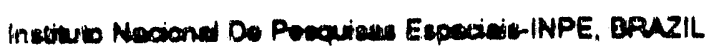

Docurnents Otros, Alomic Enorgy of Conad Lل., CANADA

Or. M.P. Bectyrraki, MPB Tectinotogies, Inc., CANADA

Dr. H.M. Skargond, Univ. of Seckatchowen, CANADA

Prot. J. Toichmam, Univ. of Montred, CANAOA

Prof. S.R. Sreonivesen, Univ. of Coloery, CANAOA

Prof. T.W. Johnetron, INRS-Energie, CANAOA

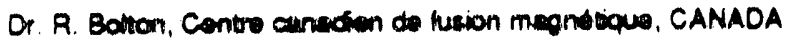

Or. C.R. Jamee., Univ. a Aberta. CANADA

Or. P. Lukte, Komonaketio Universith, CZECHOSLOVAKIA

The Libranian, Cultam Luborabry, ENGLAND

Librey, A61, Authortord Appleton Leboratory. ENGLANO

Mr. S.A. Hulchinson, JET LDYrery, ENGLAND

Dr. S.C. Shama Univ. of South Pucitic. FIJI ISLANDS

P. Menonam, Univ. of Helcinti, FINLAND

Prot. M.N. Busecec, Ecols Potyochniquis., FRANCE

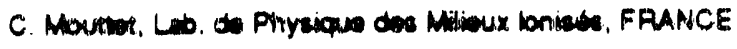

J. Radat CENCADARACHE - Bat 506, FRANCE

Prot. E. Economow, Univ. of Crow, GREECE

Me. C. Pinuri, Univ of loamina, GREECE

D. T. Mue, Acacterny Biblographic Sar., HONG KOWG

Proprint Librery. Hungainan Acaderny of SCi., HUNGAP'Y

Or. B. Descupta, Sarm inst of Nuctere Ptyrices, INDIA

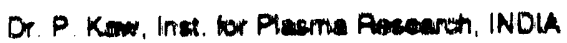

D.P. Acowew, lerad inst of Tetnology, ISPAEL

Lorangen, Intorrabiond Conser for Theo Phyeica. ITALY

Wirs C. De Puto, Aseociazion EURATOAENEA, ITALY

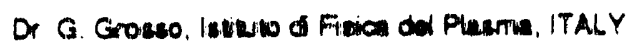

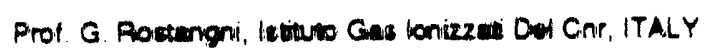

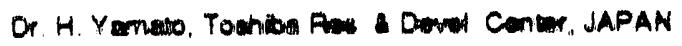

Prof. I. Kawatcemi, Hirothima Univ., JAPAN

Prof. K. Nishikewawn. Hirochima Univ., JAPAN

Director, Japen Atomic Enorgy Rosearch Irist. JAPAN

Prof. S. Itoh, Kyuthu Univ., JAPAN

Foeserch into. Cr., Nations instit. for Fusion Scionce, JAPAN

Prof. S. Tenaka, Kyolo Univ., JAPAN

Libran, Kyow Univ., JAPAN

Prot. N. Inous, Univ. of Takyo, JAPAN

Secreary, Plema Sectien, Electrolanthniced Lab. JAPAN

5. Mori, Teohni-y Advisor, LAER, MAPAN

Dr. O. Mined, Kunumots inct of Teshnology. JAPAN

J. Hycon-Sook, Korw Alomic Enory Roserch Inat, KOREA

D.1. Cha, The Kore Adv. Inst of So. \& Tech., KOREA

Prot. B.S. Lity, Univ, of Wrikato, NEW ZEALAND

Int of Phycics, Chinese Aced Sa PEOPLE'S REP. OF CHINA

Librey, Inst of Plasma Physics, PEOPLE'S REP. OF CHINA

Toinghua Univ. Lbany, PEOPLE'S AEPUBLIC OF CHINA

2. L. S.W. InA Phycics, PEOPLE'S REPUBLUC OF CHINA

Prol. J.A.C. Cabrel, instuto Superior Tecnico, PORTUGAL

Dr. O. Ponus, ML I CUzA Úniv., ROMANIA

D. J. Vilume, Fution Siucies, AEC, S. AFFICA

Prof. M.A. Helborg, Univ. of Natol, S. AFRICA

Prot. D.E. Kim. Ponang Int. of Sa. Tech., SO. KOREA

Prof. C.I.E.M.A.T, Fution Divicion Library, SPAIN

Dr. L SWmito, Univ. of UMEA, SWEDEN

Lorary, Royd inct of Technology, SWEDEN

Prol. H. Whomeon, Chamore Univ. of Tech., SWEDEN

Comtro Phys. Dos Plamms, Ecolo Patyech, SWITZERANO

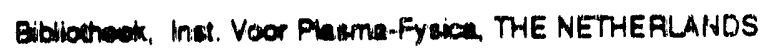

Asse Prot. Dr. S. Cakir, Windo East Tern. Univ., TUAKEY



Dr. D.D. Aputov, Siberian Enench of ACademy of Ba., USSA

D. G.A. Elienow, I.V. Kurctiator Inst, USSA

Librarien, The Ukt.SSA Academy of Sciorices. USSR

Or. LM. Kowrizhrykh, Intet of Conord Phyeics, USSA

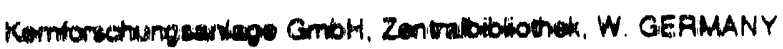

Bibliounath Inse. FOr Prusmatorschung, W. GERMANY

Prot. K. Schindar, Aurt-Univer Bochum, W. GERMANY

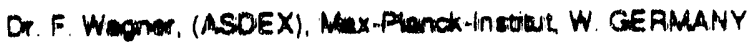

Librunan, Max-Plandx-Inother, W GERMAAYY

Prol. R.K. Jenow, Inte of Physikes. YUGOSLAVIA 

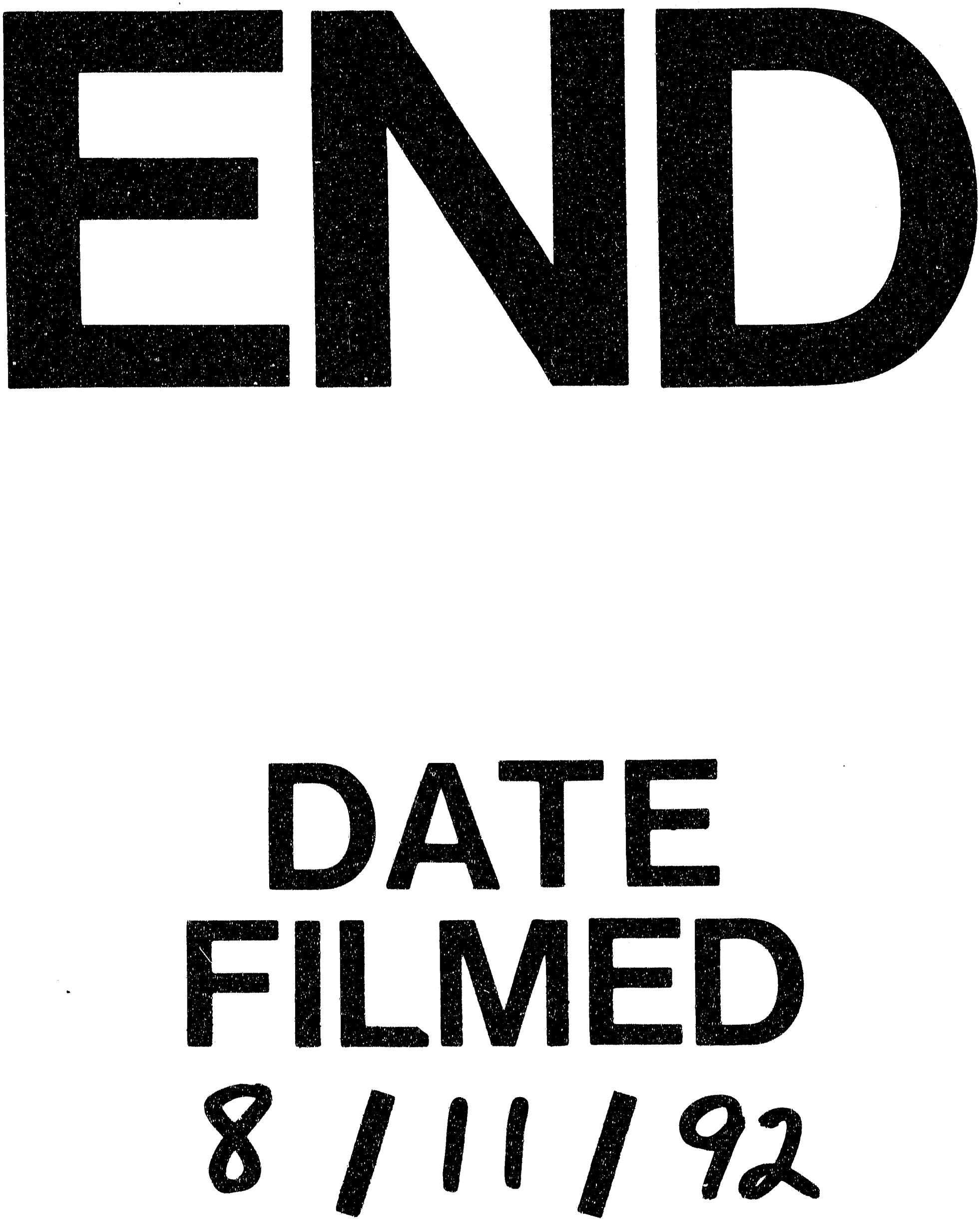


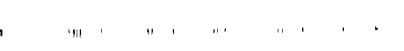

